\title{
Adseverin mediates RANKL-induced osteoclastogenesis by regulating NFATc1
}

\author{
Min-Kyoung Song, Zang Hee Lee and Hong-Hee Kim \\ Adseverin is a $\mathrm{Ca}^{2+}$-dependent actin filament-severing protein that has been reported to regulate exocytosis via rearrangements of \\ the actin cytoskeleton in secretory cells. However, the role of adseverin in bone cells has not yet been well characterized. Here, \\ we investigated the role of adseverin in osteoclastogenesis using primary osteoclast precursor cells. Adseverin expression was \\ upregulated during RANKL (receptor activator of nuclear factor-кB ligand)-induced osteoclast differentiation. Moreover, genetic \\ silencing of adseverin decreased the number of osteoclasts generated by RANKL. Adseverin knockdown also suppressed the \\ RANKL-mediated induction of nuclear factor of activated T-cell c1 (NFATc1), which is a key transcription factor in \\ osteoclastogenesis. In addition, adseverin knockdown impaired bone resorption and the secretion of bone-degrading enzymes \\ from osteoclasts. These effects were accompanied by decreased NFATc1 expression and the activation of nuclear factor- $\mathrm{kB}$. \\ Collectively, our results indicate that adseverin has a crucial role in osteoclastogenesis by regulating NFATc1. \\ Experimental \& Molecular Medicine (2015) 47, e199; doi:10.1038/emm.2015.94; published online 18 December 2015
}

\section{INTRODUCTION}

Bone is a complex tissue that is constantly remodeled and maintained by cycling between bone formation and resorption throughout life. This dynamic phenomenon is mediated by two types of cells, osteoblasts and osteoclasts. Osteoblasts form the bone matrix by depositing organic and inorganic components, whereas osteoclasts resorb bone by secreting degrading enzymes and protons. Osteoclasts are multinuclear cells that differentiate from the monocyte/macrophage lineage of hematopoietic cells. ${ }^{1}$ Two main factors are needed for osteoclast differentiation, including macrophage-colony-stimulating factor (M-CSF) and the receptor activator of nuclear factor- $\mathrm{\kappa B}$ ligand (RANKL). M-CSF is important for proliferation of the monocyte/macrophage lineage and osteoclast survival, ${ }^{2}$ whereas RANKL induces differentiation during osteoclastogenesis. ${ }^{3}$ When RANKL binds to its receptor (RANK), TNF receptorassociated factor 6 (TRAF6) is recruited, thereby initiating the nuclear factor- $\mathrm{\kappa B}$ (NF- $\mathrm{\kappa B})$ and mitogen-activated protein kinase (MAPK) signaling pathways. The calcium signaling pathway is also activated by RANKL in response to additional stimulation of surface immune receptors. These pathways all culminate in the activation of nuclear factor of activated T-cell (NFAT) c1 (NFATc1), the master transcription factor of osteoclastogenesis. Taken together with other accessory transcription factors, NFATc1 drives the expression of a number of osteoclast-specific genes. ${ }^{4}$ Upon expression of osteoclastspecific genes including tartrate-resistant acid phosphatase (TRAP), cathepsin $K$ and dendritic cell-specific transmembrane protein (DC-STAMP), osteoclasts become mature and functionally activated. Mature osteoclasts secrete bone-degrading enzymes into the resorption lacunae by exocytosis. By sealing off the resorption lacunae with an actin belt, osteoclasts induce the effective concentration of enzymes and protons in the resorbing space.

Adseverin, also called scinderin, is a member of the gelsolin superfamily of actin-binding proteins. Although adseverin has the highest degree of homology with gelsolin $(\sim 60 \%$ at the amino-acid level), the expression of adseverin is more restricted. ${ }^{5}$ Adseverin was first discovered in chromaffin cells of the adrenal medulla, and it is now believed to be expressed in all secretory cells. In secretory cells, adseverin mediates actin cytoskeletal remodeling during exocytosis. ${ }^{6-8}$ Similar to gelsolin, adseverin contains six gelsolin-like $(G)$ domains, three actin binding sites and two calcium binding sites with different affinities. $^{7,9}$ Phosphatidylinositol-4,5-bisphosphate has been shown to inhibit the interaction between actin and adseverin, most likely through competition between actin and phosphatidylinositol-4,5-bisphosphate. ${ }^{10,11}$ Upon stimulation, adseverin interacts with F-actin in the presence of $\mathrm{Ca}^{2+}$ and decreases the viscosity of actin gels by severing actin filaments. ${ }^{7}$

Department of Cell and Developmental Biology, BK21 Program and Dental Research Institute, Seoul National University School of Dentistry, Seoul, Korea Correspondence: Professor H-H Kim, Department of Cell and Developmental Biology, BK21 Program and Dental Research Institute, Seoul National University School of Dentistry, 28 Yeongon-Dong, Chongno-Gu, Seoul 110-749, Korea.

E-mail: hhbkim@snu.ac.kr

Received 23 April 2015; revised 7 September 2015; accepted 17 September 2015 
Immunofluorescence experiments have revealed that adseverin is mainly localized in the subplasmalemmal region, near a mesh of actin filaments, in chromaffin cells. ${ }^{7}$ Although the role of adseverin in chromaffin cells has been well characterized, the role of adseverin in other secretory cells, including osteoclasts, is relatively unknown. One report found that gelsolin ${ }^{-1-}$ mice exhibited increased bone thickness, high bone mass and defects in podosome assembly and bone resorption, ${ }^{12}$ suggesting that additional gelsolin superfamily proteins such as adseverin may be involved in bone remodeling.

In the present study, we found that adseverin expression is upregulated during the differentiation of primary bone marrow-derived macrophages (BMMs) into osteoclasts. Using small interfering RNA (siRNA)-mediated gene silencing, we found that adseverin promotes osteoclast differentiation by upregulating NFATc1 and activating NF- $\kappa \mathrm{B}$ signaling, especially p65 phosphorylation and nuclear translocation.

\section{MATERIALS AND METHODS}

\section{Reagents}

Recombinant human M-CSF and human soluble RANKL were purchased from PeproTech (Rocky Hill, NJ, USA). Antibodies against ERK, JNK, p38, Akt, p65, IкB and PARP were obtained from Cell Signaling Technology (Cambridge, MA, USA), as were phosphospecific antibodies for ERK (Thr202/Tyr204), JNK (Thr182/Tyr185), p38 (Thr180/Tyr182), Akt (Ser473), p65 (Ser536) and IкB (Ser32). Monoclonal antibodies against $\beta$-actin (AC-74) and secondary antibodies were obtained from Sigma-Aldrich (St Louis, MO, USA). Antibodies against adseverin (N17), NFATc1 (7A6), c-Fos (H125), lamin B (M-20) and $\alpha$-tubulin (TU-02) were purchased from Santa Cruz Biotechnology (Santa Cruz, CA, USA). The siRNA targeting adseverin and the negative control siRNA were also obtained from Santa Cruz Biotechnology. All other reagents were obtained from Sigma-Aldrich.

\section{Culture of osteoclasts}

To obtain mouse bone marrow-derived osteoclasts, 5-week-old female ICR mice were killed and their bone marrow was flushed from their tibiae and femora. After eliminating erythrocytes using hypotonic buffer, cells were incubated overnight in $\alpha$-modified Eagle's medium $(\alpha$-MEM) supplemented with $10 \%$ fetal bovine serum in culture dishes. Adherent cells were discarded, and nonadherent cells were further incubated with $30 \mathrm{ng} \mathrm{ml}^{-1} \mathrm{M}$-CSF in Petri dishes. After 3 days, adherent BMMs were collected by scraping and used as osteoclast precursor cells. BMMs were differentiated into osteoclasts by culturing them with $30 \mathrm{ng} \mathrm{ml}^{-1} \mathrm{M}$-CSF and $120 \mathrm{ng} \mathrm{ml}^{-1}$ RANKL.

\section{TRAP staining}

Cells were fixed in 3.7\% formaldehyde and permeabilized using $0.1 \%$ Triton X-100. Leukocyte acid phosphatase (TRAP) staining was performed in the dark for 5-15 min using a commercially available kit (Sigma-Aldrich) according to the manufacturer's instructions.

\section{siRNA-mediated knockdown}

BMMs were transfected with $30 \mathrm{~nm}$ adseverin-specific siRNAs (Santa Cruz Biotechnology). Briefly, siRNAs and HiPerFect (Qiagen, Hilden, Germany) or Lipofectamine 2000 (Invitrogen, Carlsbad, CA, USA) transfection reagent were mixed with serum- and antibiotic-free
$\alpha$-MEM. The siRNA mixtures were incubated for $20 \mathrm{~min}$ at room temperature and then added to the cells. The cells were incubated for $6-14 \mathrm{~h}$, and then the culture medium was replaced with $\alpha$-MEM supplemented with serum.

\section{Reverse transcription-polymerase chain reaction}

Total RNA was isolated from cells using TRIzol reagent (Invitrogen) according to the manufacturer's instructions. Next, $3 \mu \mathrm{g}$ RNA was used for cDNA synthesis using Superscript II reverse transcriptase (Invitrogen). The PCR reaction was performed in a thermal cycler (Bio-Rad, Hercules, CA, USA), and the thermocycling conditions were as follows: an initial denaturation step at $95^{\circ} \mathrm{C}$ for $5 \mathrm{~min}$, followed by 27 cycles of denaturation at $95^{\circ} \mathrm{C}$ for $30 \mathrm{~s}$, annealing at $57^{\circ} \mathrm{C}$ for $30 \mathrm{~s}$ and extension at $72^{\circ} \mathrm{C}$ for $30 \mathrm{~s}$. The following primers were used for amplification: adseverin, 5'-GAGGAACAGACCCAGCAAAT- $3^{\prime}$ and 5'-GCCACAGAGGCCTGTATCTT-3'; and HPRT, 5'-CCTAAGATG AGCGCAAGTTGAA-3' and 5'-CCACAGGGACTAGAACACCTGC TAA-3'. Real-time PCR was performed using an ABI7300 real-time system (Applied Biosystems, Warrington, UK) and a KAPA SYBR FAST qPCR Kit (Kapa Biosystems, Wilmington, MA, USA). Thermocycling conditions were as follows: an initial activation step at $95^{\circ} \mathrm{C}$ for $3 \mathrm{~min}$, followed by 40 cycles of denaturation at $95^{\circ} \mathrm{C}$ for $3 \mathrm{~s}$ and amplification at $60^{\circ} \mathrm{C}$ for $33 \mathrm{~s}$. All reactions were run in triplicate, and the housekeeping gene HPRT was used for normalization. Gene expression levels were determined as fold changes using the cycle threshold comparison method. The following primer sets were used: adseverin, 5'-TCCAGAGCAGAGAGCTTCAA- $3^{\prime}$ and 5'-TTCGCA GTCAGATCATTGGT-3'; NFATc1， 5'-CCAGTATACCAGCTCTG CCA- $3^{\prime}$ and 5'-GTGGGAAGTCAGAAGTGGGT-3'; TRAP, 5'-CGA CCATTGTTAGCCACATACG- $3^{\prime}$ and $5^{\prime}$-TCGTCCTGAAGATACTG CAGGTT-3'; cathepsin K, 5' ${ }^{\prime}$-ATATGTGGGCCACCATGAAAGTT-3' and 5'-TCGTTCCCCACAGGAATCTCT-3'; DC-STAMP, 5'-GGGTG CTGTTTGCCGCTG-3' and $5^{\prime}$-CGACTCCTTGGGTTCCTTGCT-3'; v-ATPase (Atp6v0d2), 5'-GGGAGACCCTCTTCCCCACC-3' and 5'-CCACCGACAGCGTCAAACAAA-3'; and HPRT, 5'-CCTAAG ATGAGCGCAAGTTGAA- $3^{\prime}$ and $5^{\prime}$-CCACAGGGACTAGAACACC TGCTAA-3'.

\section{Immunocytochemistry}

BMMs were cultured on 15-mm glass coverslips in 24-well plates and then fixed with $3.7 \%$ formaldehyde. After permeabilization with $0.1 \%$ Triton X-100, nonspecific binding sites were blocked using phosphatebuffered saline containing $1 \%$ bovine serum albumin for $1 \mathrm{~h}$ and $30 \mathrm{~min}$. Cells were then incubated with anti-adseverin antibodies overnight at $4{ }^{\circ} \mathrm{C}$. Cells were then washed and stained using rhodamine-phalloidin and fluorescein isothiocyanate-conjugated secondary antibodies for $2 \mathrm{~h}$. After washing the cells with phosphate-buffered saline, they were mounted in the presence of 4',6-diamidino-2-phenylindole (DAPI). Images were obtained using a ZEISS LSM700 confocal microscope (Carl Zeiss MicroImaging GmbH, Goettingen, Germany).

\section{Transwell migration assay}

BMMs were seeded in Petri dishes and transfected with adseverin and control siRNAs. After culturing the cells with $30 \mathrm{ng} \mathrm{ml}^{-1} \mathrm{M}-\mathrm{CSF}$ and $120 \mathrm{ng} \mathrm{ml}^{-1}$ RANKL for 2 days, the cells were scraped and transferred to the upper chambers of transwell plates (Corning, Corning, NY, USA). The lower chambers contained $240 \mathrm{ng} \mathrm{ml}^{-1}$ RANKL as an attractant. After $16 \mathrm{~h}$ of incubation in a $\mathrm{CO}_{2}$ incubator at $37^{\circ} \mathrm{C}$, cells were fixed and stained with crystal violet. 


\section{Resorption assay}

BMMs were seeded on calcium phosphate-coated 48-well plates and cultured with $30 \mathrm{ng} \mathrm{ml}^{-1} \mathrm{M}-\mathrm{CSF}$ and $120 \mathrm{ng} \mathrm{ml}^{-1}$ RANKL. After 7 days, the plates were rinsed with distilled water and stained with von Kossa reagents. Photographs were taken under a light microscope, and the resorption area was quantified using the ImageJ software (NIH, Bethesda, MD, USA).

\section{MMP assay}

BMMs were cultured with $30 \mathrm{ng} \mathrm{ml}^{-1} \mathrm{M}-\mathrm{CSF}$ and $120 \mathrm{ng} \mathrm{ml}^{-1}$ RANKL for 2 days, after which the medium was replaced with serum-free $\alpha$-MEM. After $12 \mathrm{~h}$, the conditioned medium was collected, and matrix metalloproteinase (MMP) activity was measured using an MMP Activity Assay Kit (Abcam, Cambridge, UK) according to the manufacturer's instructions.

\section{Western blotting}

Cells were lysed using RIPA buffer (120 mM Tris- $\mathrm{HCl}$ ( $\mathrm{pH} 7.5$ ), $150 \mathrm{~mm} \mathrm{NaCl}, 1 \mathrm{~mm} \mathrm{Na} \mathrm{EDDTA} 1 \mathrm{~mm}$ EGTA, 0.5\% NP-40, $2.5 \mathrm{~mm}$ sodium pyrophosphate, $1 \mathrm{~mm} \beta$-glycerophosphate, $1 \mathrm{~mm} \mathrm{Na}_{3} \mathrm{VO}_{4}$, $1 \mathrm{~mm} \mathrm{NaF}$ and a protease inhibitor cocktail; Roche, Mannheim, Germany). The protein concentrations of the cell lysates were measured using a DC Protein Assay Kit (Bio-Rad), and equal amounts of protein were loaded onto 8 or $10 \%$ sodium dodecyl sulfate-polyacrylamide gels for electrophoresis. After transfer onto nitrocellulose membranes (Amersham Pharmacia, Uppsala, Sweden), nonspecific binding sites on the membranes were blocked with 5\% non-fat skim milk for $1 \mathrm{~h}$, and the membranes were then incubated with primary antibodies overnight at $4{ }^{\circ} \mathrm{C}$. The membranes were then washed several times with TBST (Tris-buffered saline with Tween-20) and incubated with horseradish peroxidase-conjugated secondary antibodies in $2 \%$ skim milk for $1 \mathrm{~h}$. Immunoreactive bands were detected using ECL reagents in the dark. Nuclear and cytoplasmic protein fractions were prepared using NE-PER nuclear and
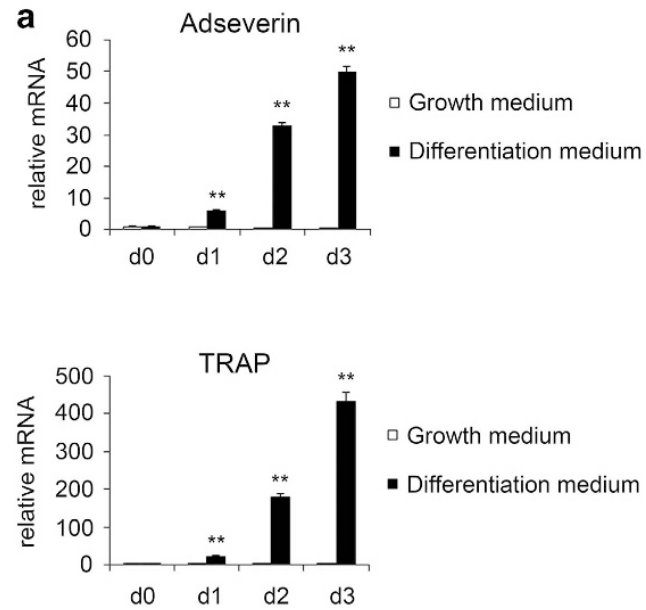

cytoplasmic extraction reagents (Pierce, Rockford, IL, USA) according to the manufacturer's protocols.

\section{Statistical analysis}

Statistical differences between results were tested using Student's $t$-tests. $P$-values $<0.05$ were regarded as significant.

\section{RESULTS}

Adseverin expression is upregulated during RANKL-induced osteoclastogenesis

In our preliminary studies, we used microarrays to identify the transcriptional changes that accompany osteoclast differentiation. We found that adseverin expression was upregulated 12-fold at day 3 after RANKL treatment (data not shown). To validate this result, BMMs were cultured with M-CSF and RANKL, and the level of adseverin mRNA was measured using quantitative real-time PCR. Adseverin expression was found to be increased up to 50-fold at day 3 (Figure 1a). Upregulation of $T R A P$, an osteoclastogenesis marker gene, was also detected, confirming that effective osteoclast differentiation occurred in the primary precursor cells. Moreover, RANKL treatment increased the level of adseverin mRNA in a dose-dependent manner at concentrations up to $120 \mathrm{~nm}$ (Figure 1b). The level of adseverin protein was also increased, peaking at day 3 post-RANKL treatment (Figure 1c).

\section{Adseverin depletion impairs osteoclast differentiation and actin ring formation}

To investigate whether adseverin has a role in osteoclastogenesis, adseverin expression was silenced in BMMs using adseverin-specific siRNAs (Figure 2a). As expected, culturing control cells with M-CSF and RANKL resulted in a

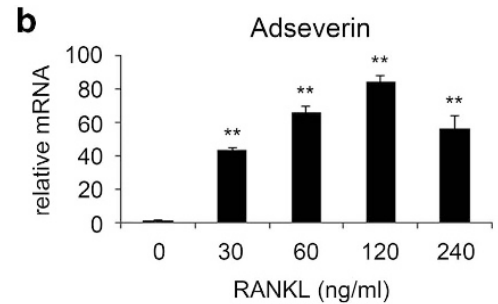

C

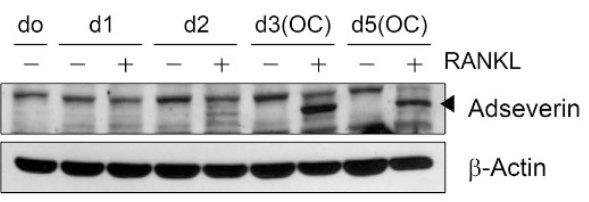

Figure 1 The expression of adseverin during receptor activator of nuclear factor- $\mathrm{KB}$ ligand (RANKL)-induced osteoclastogenesis. (a) Bone marrow-derived macrophages (BMMs) were cultured with $30 \mathrm{ng} \mathrm{ml}^{-1}$ macrophage-colony-stimulating factor (M-CSF) and $120 \mathrm{ng} \mathrm{ml}^{-1}$ RANKL for 0, 1, 2 or 3 days. Total RNA was isolated and analyzed using quantitative real-time PCR. The mRNA level of adseverin was normalized to the level of HPRT (hypoxanthine-guanine phosphoribosyltransferase). The mRNA level of tartrate-resistant acid phosphatase (TRAP), a marker of osteoclast differentiation, was also analyzed. (b) BMMs were stimulated with the indicated doses of RANKL in the presence of $30 \mathrm{ng} \mathrm{ml}^{-1} \mathrm{M}$-CSF. Cells were cultured for 3 days and then analyzed using real-time PCR. (c) Western blot analysis was performed using antibodies against adseverin and $\beta$-actin. Protein lysates were prepared from BMMs cultured for $0,1,2,3$ or 5 days with $30 \mathrm{ng} \mathrm{ml}^{-1} \mathrm{M}-\mathrm{CSF}$ and $120 \mathrm{ng} \mathrm{ml}^{-1}$ RANKL. The data are presented as the means \pm s.d. ${ }^{* *} P<0.005$. 
a

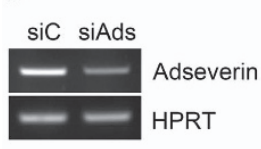

C

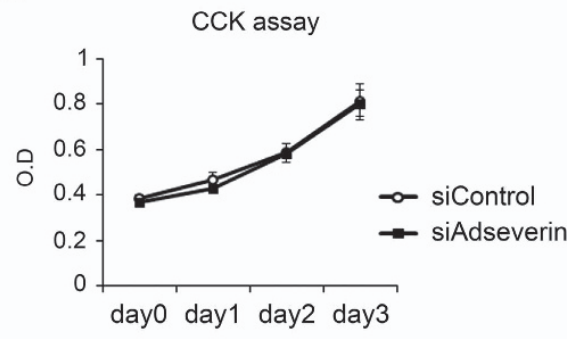

e

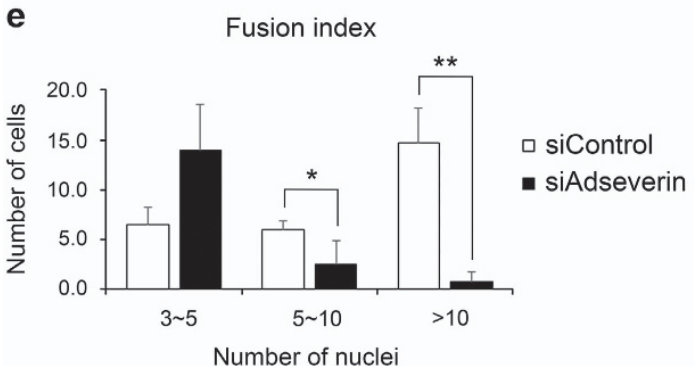

b siControl

d
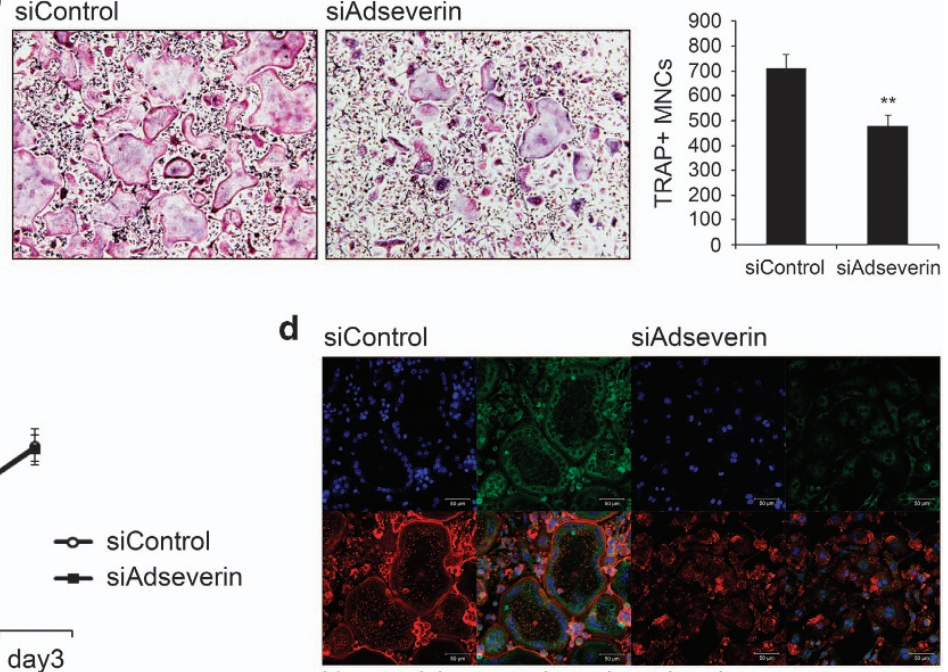

blue, nuclei; geen, adseverin; red, actin

f sicontrol

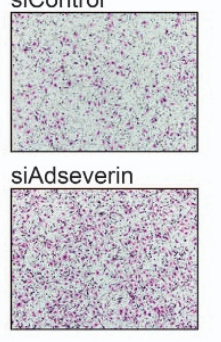

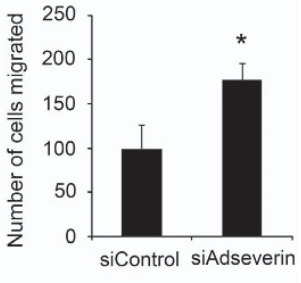

Figure 2 Adseverin depletion impairs osteoclast differentiation and actin ring formation. (a) Bone marrow-derived macrophages (BMMs) were transfected with $30 \mathrm{~nm}$ of control or adseverin-specific small interfering RNA (siRNA) and cultured for 2 days with $120 \mathrm{ng} \mathrm{ml}^{-1}$ receptor activator of nuclear factor- $\mathrm{KB}$ ligand (RANKL) and $30 \mathrm{ng} \mathrm{ml}^{-1}$ macrophage-colony-stimulating factor (M-CSF). Adseverin mRNA expression was then analyzed using reverse transcription-polymerase chain reaction (RT-PCR). (b) siRNA-transfected BMMs were cultured with M-CSF and RANKL for 4 days. Cells were fixed and then stained for tartrate-resistant acid phosphatase (TRAP). Images were captured using a light microscope (magnification $\times 100$ ), and TRAP-positive multinucleated cells $($ MNCs) were counted. (c) siRNA-transfected BMMs were cultured in 96-well plates. CCK assays were performed on days 0, 1, 2 and 3 post-RANKL treatment. (d) BMMs were cultured with $30 \mathrm{ng} \mathrm{ml}^{-1} \mathrm{M}-\mathrm{CSF}$ and $120 \mathrm{ng} \mathrm{ml}^{-1}$ RANKL for 4 days. The actin ring was labeled with rhodamine-phalloidin (red), and cells were also immunostained using anti-adseverin-FITC (fluorescein isothiocyanate) antibodies. Nuclei were counterstained with 4',6-diamidino-2phenylindole (DAPI). (e) As in d, fixed cells were stained with DAPI and rhodamine-phalloidin and then counted for the number of nuclei per osteoclast. (f) BMMs were cultured for 2 days with M-CSF and RANKL, and migration assays were performed for $16 \mathrm{~h}$ using transwell plates. The data are presented as the means \pm s.d. ${ }^{*} P<0.05 ; * * P<0.005$.

considerable number of multinucleated cells that were positive for the osteoclast marker TRAP at day 4. However, only a few multinucleated cells were observed in the adseverin-knockdown cells; moreover, most of the multinucleated cells that were formed were smaller and harbored fewer nuclei than were observed in the control cells (Figure 2b). To examine whether adseverin controls osteoclast differentiation by regulating the proliferation of osteoclast precursors, a cell proliferation assay was performed. However, no significant difference was observed between adseverinknockdown cells and control cells (Figure 2c). Although adseverin has been reported to exert an anticancer effect by inhibiting cell proliferation, ${ }^{13}$ adseverin does not appear to affect the proliferation of osteoclast precursors.

Next, we assessed actin ring formation. The actin ring is a typical morphological feature and a functionally important cytoskeletal structure in mature osteoclasts. In control cells, actin rings clearly formed around the plasma membranes, and adseverin was primarily located in the subplasmalemmal region near the actin ring (Figure 2d). However, the actin belt was rarely observed in the adseverin-knockdown cells, and most of these cells had only one or two nuclei (Figure $2 \mathrm{~d}$ ). Because cell fusion is a process that is characteristic of osteoclastogenesis, we analyzed the fusion index by counting the number of nuclei per osteoclast. As shown in Figure 2e, adseverin knockdown led to a lower fusion index compared with the index observed in the control knockdown cells. Finally, we examined whether cell migration ability was affected by adseverin knockdown. Adseverin-knockdown cells showed a higher capacity to migrate compared with control cells (Figure 2f), suggesting that adseverin-knockdown cells might have less time to be in contact with neighboring cells for fusion. Taken together, these 
results indicate that adseverin positively regulates osteoclast differentiation, fusion and actin ring formation without affecting proliferation.

\section{Adseverin-knockdown osteoclasts display defects in resorption and enzyme secretion}

Given the morphological defects that were observed in adseverin-knockdown osteoclasts, we next examined osteoclast function in these cells. To resorb bone, osteoclasts secrete enzymes such as TRAP, cathepsin K and MMPs. We predicted that resorption activity would also be impaired in the adseverin-knockdown osteoclasts because of their clearly defective multinucleation and actin ring formation. To test this hypothesis, BMMs were cultured on calcium phosphatecoated plates, which mimic the inorganic part of the bone. At day 7, the remaining calcium phosphate was stained using von Kossa reagents. This experiment revealed that adseverinknockdown osteoclasts were defective in calcium phosphate resorption. Specifically, the knockdown cells exhibited $\sim 80 \%$ less resorption compared with control cells (Figure 3a). Next, we examined enzyme secretion from osteoclasts using bone particles to mimic the resorption environment. ${ }^{14}$ The levels of TRAP and cathepsin $\mathrm{K}$ in the conditioned medium collected from control cells increased over time; however, conditioned medium from adseverin-knockdown osteoclasts showed lower levels of these enzymes (Figure 3b). Moreover, the amount of activated MMPs in the conditioned medium of adseverin-knockdown cells was $\sim 63 \%$ lower compared with the amount in the medium of control cells (Figure 3c). This finding suggests that adseverin regulates osteoclast function by modulating the secretion of bone-degrading enzymes.

\section{NFATc1 expression and nuclear translocation are regulated by adseverin}

NFATc1 is a key transcription factor during osteoclastogenesis, and its expression is strongly induced by RANKL. ${ }^{15}$ Furthermore, NFATc1 expression is autoamplified, and NFATcl also mediates the expression of many osteoclastspecific genes. ${ }^{16}$ To investigate whether adseverin regulates NFATc1 expression during RANKL-induced osteoclastogenesis, we determined the expression level of NFATc1 in adseverinknockdown BMMs. After RANKL stimulation, the protein level of NFATc1 was significantly lower in the knockdown cells compared with that in control cells (Figure 4a). c-Fos, which is a transcription factor required for the induction of NFATc1, regulates the transcription of osteoclast-specific genes through NFATc1. ${ }^{4,15}$ c-Fos was also decreased at day 1 (Figure 4a). Adseverin is therefore likely to regulate NFATc1 expression at least, in part, by modulating of c-Fos expression. Because NFATc1 autoregulates its own expression, a decrease in NFATc1 expression is expected to be accompanied by a decrease in NFATc1 nuclear translocation. Therefore, we assessed the nuclear translocation of NFATc1 following adseverin knockdown. As expected, both nuclear and cytoplasmic NFATc1 were decreased in adseverin-knockdown cells
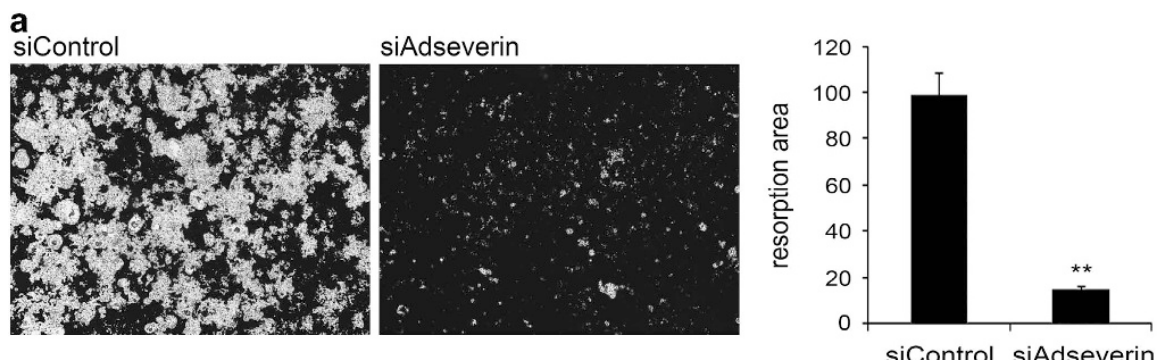

b

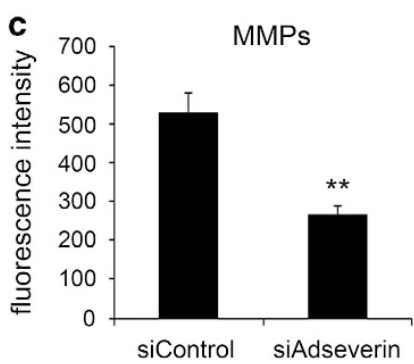

Figure 3 Adseverin-knockdown osteoclasts display defects in resorption and enzyme secretion. (a) Bone marrow-derived macrophages (BMMs) were seeded on calcium phosphate-coated 48-well plates. Cells were transfected with 30 nm adseverin small interfering RNA (siRNA) and then cultured with macrophage-colony-stimulating factor (M-CSF) and receptor activator of nuclear factor-kB ligand (RANKL) for 7 days. After the cells were washed, von Kossa staining was performed, and the resorption areas were analyzed. (b) After siRNA transfection, BMMs were cultured with M-CSF and RANKL for 2 days. The cells were further incubated in serum-free $\alpha$-modified Eagle's medium ( $\alpha-M E M)$ with bone particles obtained by grinding dentin. Supernatants were collected after 1,6 and $12 \mathrm{~h}$ of incubation and analyzed using western blot analysis with anti-tartrate-resistant acid phosphatase (TRAP) and anti-cathepsin $\mathrm{K}$ antibodies. (c) As in b, supernatants were collected from cells cultured without bone particles for $12 \mathrm{~h}$. Total matrix metalloproteinase (MMP) activity was measured using a commercial MMP Assay Kit. The data are presented as the means \pm s.d. ${ }^{* *} P<0.005$. 

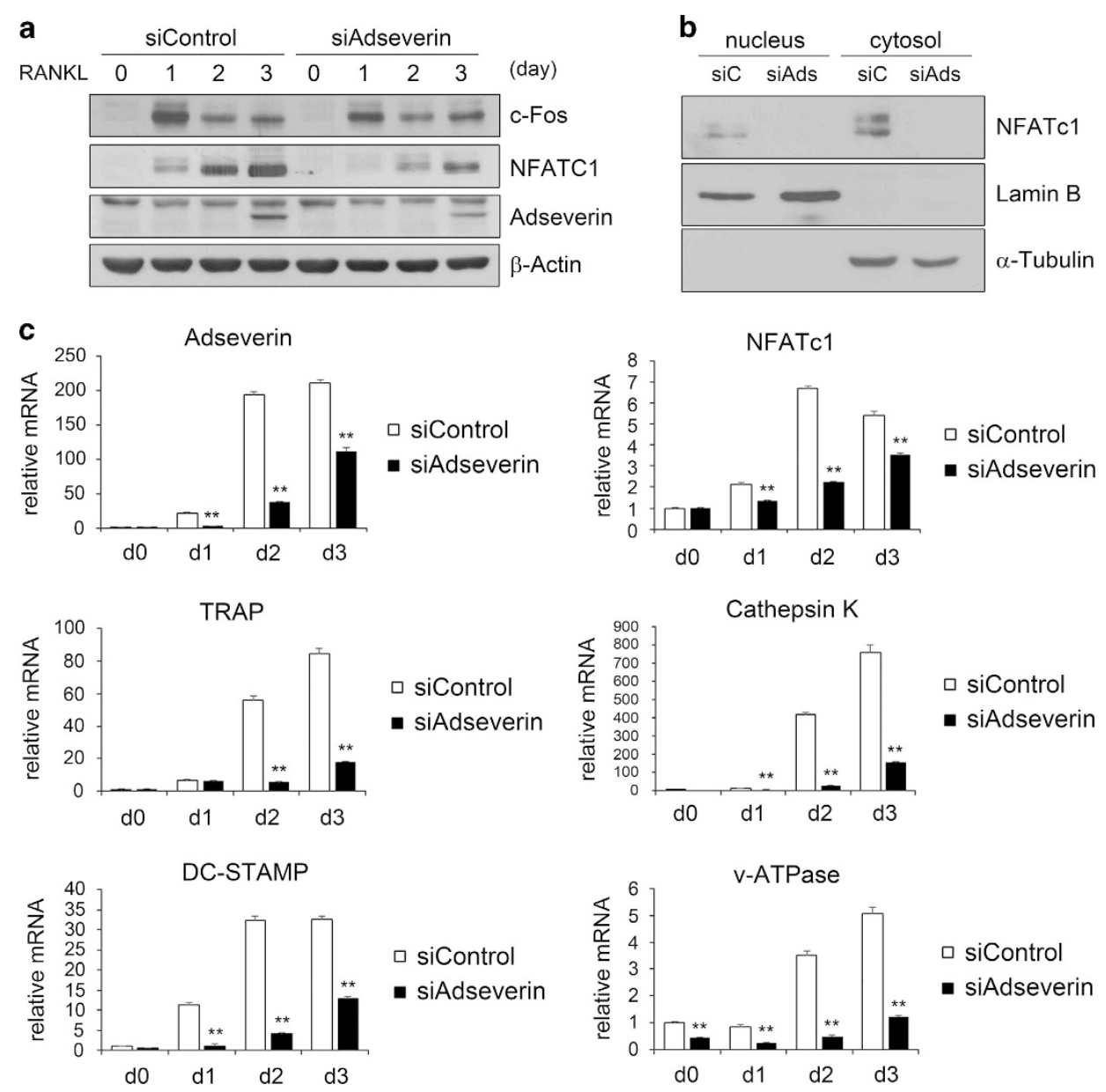

Figure 4 Adseverin regulates nuclear factor of activated T cell c1 (NFATc1) expression and nuclear translocation. (a) Protein lysates were prepared from macrophage-colony-stimulating factor (M-CSF)/receptor activator of nuclear factor-kB ligand (RANKL)-treated bone marrowderived macrophages (BMMs) after small interfering RNA (siRNA) transfection. Western blot analysis was performed using antibodies for c-Fos, NFATc1 and adseverin. (b) After siRNA transfection, BMMs were cultured for 2 days in the presence of M-CSF and RANKL. Nuclear and cytoplasmic fractions were prepared and analyzed using western blot analysis. Lamin B and $\alpha$-tubulin were used as controls for nuclear and cytoplasmic proteins, respectively. (c) BMMs were transfected with adseverin siRNA and then cultured with M-CSF and RANKL for 3 days. The expression levels of adseverin, NFATc1, tartrate-resistant acid phosphatase (TRAP), cathepsin K, dendritic cellspecific transmembrane protein (DC-STAMP) and v-ATPase were analyzed using real-time PCR. The data are presented as the means \pm s.d. $* * P<0.005$.

(Figure 4b). Moreover, the expression of osteoclast-specific genes that are directly induced by NFATc1, such as TRAP, cathepsin K, DC-STAMP and v-ATPase, were also markedly decreased during osteoclast differentiation in adseverindeficient cells compared with control cells (Figure 4c). These results indicate that adseverin regulates osteoclast differentiation by modulating the expression of c-Fos and NFATc1, and, consequently, the expression of NFATc1-driven genes.

\section{Adseverin regulates osteoclastogenesis via the NF-кB} signaling pathway and not the MAPK signaling pathway To investigate the mechanisms by which adseverin regulates NFATc1 expression during RANKL-induced osteoclastogenesis, we examined the effects of adseverin knockdown on the MAPK signaling pathway. MAPKs have been reported to be activated by RANKL and to upregulate NFATc1 expression through AP-1. After elevating adseverin expression by culturing cells with RANKL for 2 days, the activation of MAPK signaling proteins was assessed using western blot analysis with phosphospecific MAPK antibodies. RANKL-mediated activation of ERK, JNK and p38 was observed in both the adseverinknockdown cells and the control cells at similar levels (Figure 5a). Next, Akt and NF-אB activation were determined. There was no difference in RANKL-induced Akt phosphorylation between the adseverin-knockdown and the control cells (Figure $5 b$ ). The canonical NF- $\kappa B$ signaling pathway, which involves the phosphorylation and degradation of I $\mathrm{KB}$ and the subsequent nuclear translocation of NF- $\kappa \mathrm{B}$, was activated by RANKL. There was no clear distinction between the adseverinknockdown and control cells (Figure 5c). However, RANKLinduced phosphorylation of p65 NF- $\mathrm{KB}$ was significantly reduced (Figure $5 \mathrm{c}$ ). The nuclear translocation of p65 was also decreased in the adseverin-knockdown cells compared with the control cells (Figure $5 \mathrm{~d}$ ). NF- $\kappa \mathrm{B}$ is a key signaling molecule that 

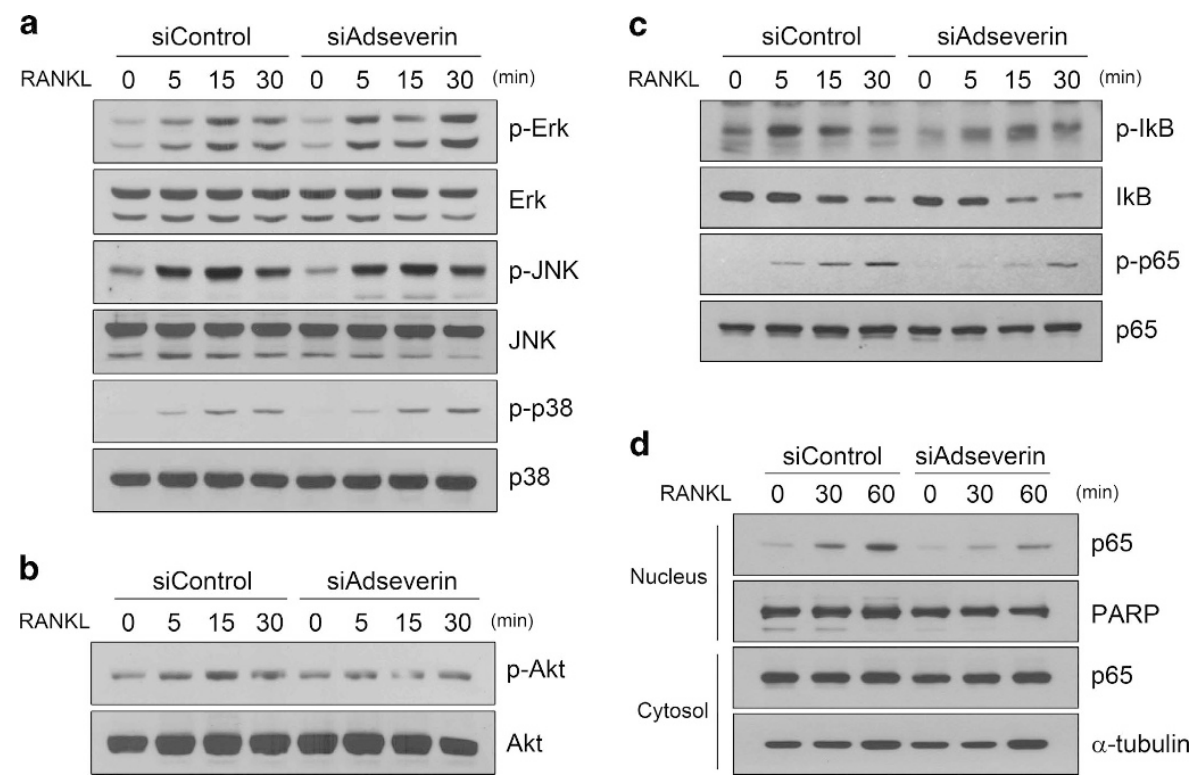

Figure 5 Adseverin regulates osteoclastogenesis via the nuclear factor- $\kappa B(N F-\kappa B)$ signaling pathway but not the mitogen-activated protein kinase (MAPK) signaling pathway. (a-c) After small interfering RNA (siRNA) transfection, bone marrow-derived macrophages (BMMs) were cultured with $30 \mathrm{ng} \mathrm{ml}^{-1}$ macrophage-colony-stimulating factor (M-CSF) and $120 \mathrm{ng} \mathrm{ml}^{-1}$ receptor activator of nuclear factor-kB ligand (RANKL) for 2 days. Cells were serum-starved for $4 \mathrm{~h}$ in $\alpha$-modified Eagle's medium ( $\alpha$-MEM) and then stimulated with $600 \mathrm{ng}^{-1}$ RANKL. Stimulated cells were harvested after 0, 5, 15 and 30 min, and protein lysates were then prepared. Western blot analysis was performed using antibodies against the phosphoforms or total forms of the indicated proteins. (d) Cells were stimulated with RANKL as in a-c for 0,30 and $60 \mathrm{~min}$. Nuclear and cytoplasmic fraction proteins were prepared and subjected to western blot analysis.

is activated by RANKL that is known to regulate NFATc1 expression in osteoclastogenesis. ${ }^{4,16}$ Therefore, these data demonstrate that adseverin has a role in RANKL-induced osteoclastogenesis by regulating the noncanonical NF- $\kappa B$ signaling pathway but not the MAPK signaling pathway.

\section{DISCUSSION}

$\mathrm{Ca}^{2+}$ signaling is one of the main intracellular events that is involved in osteoclast differentiation. Adseverin is a $\mathrm{Ca}^{2+}$ regulated protein that has been shown to have $\mathrm{Ca}^{2+}$-dependent actin-severing activity. Based on these observations, we hypothesized that adseverin has a role in osteoclast differentiation. We found that adseverin expression was increased during osteoclast differentiation (Figure 1). Furthermore, our results provide evidence showing that adseverin controls osteoclast differentiation by regulating NFATc1 expression and NF- $\mathrm{KB}$ signaling (Figures 4 and 5).

$\mathrm{NF}-\mathrm{\kappa B}$ has been demonstrated to have a crucial role in osteoclastogenesis. The p50 and p65 NF- $\mathrm{kB}$ subunits are recruited to the NFATc1 promoter in response to RANKL stimulation. ${ }^{16}$ In addition, dehydroxymethylepoxyquinomicin, an NF- $\mathrm{BB}$ inhibitor, has been shown to attenuate RANKLinduced osteoclastogenesis by downregulating NFATc1. ${ }^{17}$

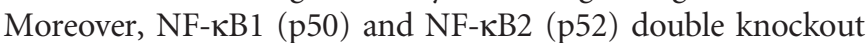
mice display a severe osteopetrotic phenotype. ${ }^{18}$ Therefore, NF- $\kappa \mathrm{B}$ has a crucial role in osteoclastogenesis by mediating NFATc1 expression. We observed that p65 phosphorylation was reduced following adseverin knockdown (Figure $5 \mathrm{c}$ ). Because p65 phosphorylation enhances the transactivation potential of NF-KB, ${ }^{19,20}$ it is likely that adseverin regulates
NF- $\mathrm{KB}$ transcription activity during osteoclastogenesis. In addition, we found that the nuclear translocation of p65 was regulated by adseverin (Figure 5d). However, there was no difference indicating its involvement in IкB degradation (Figure 5c), which excludes the possibility of adseverinmediated regulation of the canonical NF- $\mathrm{kB}$ pathway. Sasaki et al. ${ }^{21}$ reported a noncanonical NF- $\mathrm{KB}$ pathway in which the nuclear translocation of phosphorylated p65 was independent of regulation by IкB. Taken together, these data indicate that adseverin regulates p65 phosphorylation and nuclear translocation in a noncanonical manner during RANKL-induced osteoclast differentiation.

Adseverin is known to be expressed in most secretory cells and is believed to have a role in actin cytoskeletal rearrangements during exocytosis. In osteoclasts, numerous vesicles containing lysosomal enzymes are transported to the cell surface, and their contents are released by exocytosis. ${ }^{22}$ These released enzymes degrade the organic components of the bone matrix. Consistent with this role of adseverin in exocytosis, we observed that adseverin knockdown reduced the secretion of bone-degrading enzymes by osteoclasts (Figure 3). This reduction in secretion is likely due to the effect of adseverin knockdown on actin cytoskeleton dynamics, in addition to the downregulation of NFATc1 levels, which reduces the expression of these enzymes.

Adseverin and gelsolin share a common function, the severing of actin filaments, and they also have high homology. Consistent with a report showing that gelsolin-knockout mice have high bone mass and display defects in osteoclast podosome assembly, ${ }^{12}$ we found that adseverin-knockdown 
osteoclasts possessed numerous defects in their differentiation and function (Figures 2 and 3). Although adseverin and gelsolin have some common features, they also have a number of different properties. Importantly, adseverin and gelsolin have different $\mathrm{Ca}^{2+}$-binding affinities. ${ }^{7}$ This finding may explain why adseverin is more specialized for exocytosis and why it is expressed in fewer tissues than gelsolin. Therefore, it is possible that gelsolin and adseverin regulate bone metabolism via different mechanisms. Gelsolin is known to regulate bone mass by affecting osteoclast podosome assembly, whereas adseverin may also regulate osteoclasts by acting as a signal transducer during the early stage of differentiation. In support of this hypothesis, we observed that NFATc1 expression was decreased at one day post-RANKL treatment (Figure 4).

Recently, actin as demonstrated to be present in both the nucleus and the cytoplasm. Furthermore, the functions of several nuclear actin-binding proteins have been identified. Many members of the gelsolin superfamily, such as flightless I, supervillin and gelsolin, which are present in the nucleus, are coactivators of nuclear receptors. Supervillin promotes the transactivation of the androgen receptor, ${ }^{23,24}$ whereas gelsolin facilitates the nuclear translocation of the androgen receptor by acting as a coregulator. ${ }^{25}$ In addition, mounting evidence supports a role for gelsolin as an upstream factor in many signaling cascades. For example, gelsolin cleaves caspase-3, the products of which are effectors of apoptosis. ${ }^{26}$ Moreover, gelsolin has been shown to regulate collagen phagocytosis via Rac-dependent control of the cytoskeleton. ${ }^{27}$ Although the potential roles of adseverin as a signal transducer or transcriptional regulator have not yet been clearly defined, some reports have concluded that adseverin affects signaltransduction pathways either directly or indirectly via cytoskeletal rearrangements and that adseverin regulates the differentiation of chondrocytes and megakaryoblastic leukemia cells. $^{28,29}$ These reports, along with data from other gelsolins, raise the possibility that adseverin may have additional functions beyond its well-known role in regulating actin polymerization in the cytoplasm.

The data presented here demonstrate that adseverin has a role in osteoclastogenesis. Specifically, adseverin regulates NFATc1 expression via NF- $\kappa \mathrm{B}$ signaling, which is necessary for effective osteoclast differentiation and resorption. This study is the first to demonstrate a role for adseverin beyond its role in controlling the actin cytoskeleton. Our data thus identify a novel role for adseverin in signal transduction and osteoclastogenesis.

\section{CONFLICT OF INTEREST}

The authors declare no conflict of interest.

\section{ACKNOWLEDGEMENTS}

This work was supported by a grant from the National Research Foundation of Korea (NRF-2014R1A2A1A10050406) to H-HK.
1 Hayashi S, Yamane T, Miyamoto A, Hemmi H, Tagaya H, Tanio Y et al. Commitment and differentiation of stem cells to the osteoclast lineage. Biochem Cell Biol 1998; 76: 911-922.

2 Valledor AF, Borras FE, Cullell-Young M, Celada A. Transcription factors that regulate monocyte/macrophage differentiation. J Leukoc Biol 1998; 63: 405-417.

3 Yasuda H, Shima N, Nakagawa N, Yamaguchi K, Kinosaki M, Mochizuki $S$ et al. Osteoclast differentiation factor is a ligand for osteoprotegerin/osteoclastogenesis-inhibitory factor and is identical to TRANCE/RANKL. Proc Natl Acad Sci USA 1998; 95: 3597-3602.

$4 \mathrm{Kim} \mathrm{JH}$, Kim N. Regulation of NFATc1 in osteoclast differentiation. J Bone Metab 2014; 21: 233-241.

5 Kwiatkowski DJ. Functions of gelsolin: motility, signaling, apoptosis, cancer. Curr Opin Cell Biol 1999; 11: 103-108.

6 Silacci P, Mazzolai L, Gauci C, Stergiopulos N, Yin HL, Hayoz D. Gelsolin superfamily proteins: key regulators of cellular functions. Cell Mol Life Sci 2004; 61: 2614-2623.

7 Rodriguez Del Castillo A, Lemaire S, Tchakarov L, Jeyapragasan M, Doucet JP, Vitale ML et al. Chromaffin cell scinderin, a novel calciumdependent actin filament-severing protein. EMBO J 1990; 9: 43-52.

8 Tchakarov L, Vitale ML, Jeyapragasan M, Rodriguez Del Castillo A, Trifaro JM. Expression of scinderin, an actin filament-severing protein, in different tissues. FEBS Lett 1990; 268: 209-212.

9 Chumnarnsilpa S, Lee WL, Nag S, Kannan B, Larsson M, Burtnick LD et al. The crystal structure of the C-terminus of adseverin reveals the actin-binding interface. Proc Natl Acad Sci USA 2009; 106: 13719-13724.

10 Maekawa S, Sakai H. Inhibition of actin regulatory activity of the 74-kDa protein from bovine adrenal medulla (adseverin) by some phospholipids. J Biol Chem 1990; 265: 10940-10942.

11 Zhang L, Marcu MG, Nau-Staudt K, Trifaro JM. Recombinant scinderin enhances exocytosis, an effect blocked by two scinderin-derived actinbinding peptides and PIP2. Neuron 1996; 17: 287-296.

12 Chellaiah M, Kizer N, Silva M, Alvarez U, Kwiatkowski D, Hruska KA Gelsolin deficiency blocks podosome assembly and produces increased bone mass and strength. J Cell Biol 2000; 148: 665-678.

13 Wang D, Sun SQ, Yu YH, Wu WZ, Yang SL, Tan JM. Suppression of SCIN inhibits human prostate cancer cell proliferation and induces GO/G1 phase arrest. Int J Oncol 2014; 44: 161-166.

14 Cremasco V, Decker CE, Stumpo D, Blackshear PJ, Nakayama KI, Nakayama $\mathrm{K}$ et al. Protein kinase $\mathrm{C}$-delta deficiency perturbs bone homeostasis by selective uncoupling of cathepsin $\mathrm{K}$ secretion and ruffled border formation in osteoclasts. J Bone Miner Res 2012; 27: 2452-2463.

15 Takayanagi H, Kim S, Koga T, Nishina H, Isshiki M, Yoshida $\mathrm{H}$ et al. Induction and activation of the transcription factor NFATc1 (NFAT2) integrate RANKL signaling in terminal differentiation of osteoclasts. Dev Cell 2002; 3: 889-901.

16 Asagiri M, Sato K, Usami T, Ochi S, Nishina H, Yoshida $\mathrm{H}$ et al. Autoamplification of NFATc1 expression determines its essential role in bone homeostasis. J Exp Med 2005; 202: 1261-1269.

17 Takatsuna H, Asagiri M, Kubota T, Oka K, Osada T, Sugiyama C et al. Inhibition of RANKL-induced osteoclastogenesis by (-)-DHMEQ, a novel NF-kappaB inhibitor, through downregulation of NFATc1. J Bone Miner Res 2005; 20: 653-662.

18 lotsova V, Caamano J, Loy J, Yang Y, Lewin A, Bravo R. Osteopetrosis in mice lacking NF-kappaB1 and NF-kappaB2. Nat Med 1997; 3: 1285-1289.

19 Sakurai H, Chiba H, Miyoshi H, Sugita T, Toriumi W. IkappaB kinases phosphorylate NF-kappaB p65 subunit on serine 536 in the transactivation domain. J Biol Chem 1999; 274: 30353-30356.

20 Doyle SL, Jefferies CA, O'Neill LA. Bruton's tyrosine kinase is involved in p65-mediated transactivation and phosphorylation of p65 on serine 536 during NFkappaB activation by lipopolysaccharide. J Biol Chem 2005; 280: 23496-23501.

21 Sasaki CY, Barberi TJ, Ghosh P, Longo DL. Phosphorylation of RelA/p65 on serine 536 defines an I\{kappa\}B\{alpha\}-independent NF-\{kappa\}B pathway. J Biol Chem 2005; 280: 34538-34547.

22 Vaananen HK, Zhao H, Mulari M, Halleen JM. The cell biology of osteoclast function. J Cell Sci 2000; 113(Pt 3): 377-381.

23 Sampson ER, Yeh SY, Chang HC, Tsai MY, Wang X, Ting HJ et al. Identification and characterization of androgen receptor associated coregulators in prostate cancer cells. J Biol Regul Homeost Agents 2001; 15: $123-129$ 
24 Ting HJ, Yeh S, Nishimura K, Chang C. Supervillin associates with androgen receptor and modulates its transcriptional activity. Proc Natl Acad Sci USA 2002; 99: 661-666.

25 Nishimura K, Ting HJ, Harada Y, Tokizane T, Nonomura N, Kang HY et al. Modulation of androgen receptor transactivation by gelsolin: a newly identified androgen receptor coregulator. Cancer Res 2003; 63 : 4888-4894.

26 Geng YJ, Azuma T, Tang JX, Hartwig JH, Muszynski M, Wu Q et al. Caspase-3-induced gelsolin fragmentation contributes to actin cytoskeletal collapse, nucleolysis, and apoptosis of vascular smooth muscle cells exposed to proinflammatory cytokines. Eur J Cell Biol 1998; 77: 294-302.

27 Arora PD, Glogauer M, Kapus A, Kwiatkowski DJ, McCulloch CA. Gelsolin mediates collagen phagocytosis through a rac-dependent step. $\mathrm{Mol} B$ iol Cell 2004; 15: 588-599.

28 Nurminsky D, Magee C, Faverman L, Nurminskaya M. Regulation of chondrocyte differentiation by actin-severing protein adseverin. Dev Biol 2007; 302: 427-437
29 Zunino R, Li Q, Rose SD, Romero-Benitez MM, Lejen T, Brandan NC et al. Expression of scinderin in megakaryoblastic leukemia cells induces differentiation, maturation, and apoptosis with release of plateletlike particles and inhibits proliferation and tumorigenesis. Blood 2001; 98: 2210-2219.

cc) (i) $(\Theta$ This work is licensed under a Creative Commons Attribution-NonCommercial-NoDerivs 4.0 International License. The images or other third party material in this article are included in the article's Creative Commons license, unless indicated otherwise in the credit line; if the material is not included under the Creative Commons license, users will need to obtain permission from the license holder to reproduce the material. To view a copy of this license, visit http://creativecommons.org/licenses/by-nc-nd/4.0/ 